\title{
Prosthetic Rehabilitation of Cranial Defects: An Overview
}

\author{
Sunil K Mishra ${ }^{1}$, Ramesh Chowdhary ${ }^{2}$ \\ International Journal of Prosthodontics and Restorative Dentistry (2019): 10.5005/jp-journals-10019-1236
}

Dating back to 3000 BC, cranioplasty is found to be one of the oldest neurosurgical procedures followed for treating cranial defects. Defects resulted in cranial vault may be a result of trauma, meningiomas, loss of a bone flap owing to infection or decompressive craniectomies and/or congenital deformities. Owing to the cranial defect, patient's physical appearance may be compromised along with unprotected brain with transmission of pulsation and vibrations from the brain, which raises a serious concern. A large cranial defect may present neurological symptoms such as discomfort, irritability, dizziness, headache, epilepsy, and psychiatric symptoms. In an attempt to overcome these problems, rehabilitation of the cranial defect is required (which is termed as cranioplasty), and it involves a multidisciplinary approach, involving neurosurgeons, craniofacial surgeons, and a maxillofacial prosthodontist. Cranioplasty helps in improving the anatomic accuracy, esthetic contouring, and neurologic function, with improvement in the cerebral blood flow, glucose metabolism, and cerebrovascular reserve capacity. ${ }^{1,2}$

Various materials have been used for centuries to cover the bony defects; these include shells of coconut, alloplastic and autologous bone grafts, resins, titanium, hydroxyapatite, silicones, ceramics, and polyether ether ketone (PEEK). The main requirement of plastic materials is the preoperative shaping for proper adaptation to the defect. An ideal requirement of a cranioplasty material is that it should be inexpensive, shapable, and durable, have a low rate of infection, and be a poor conductor of heat, nonmagnetic, radiolucent and acceptable to the tissue. The perforations are incorporated into the cranial prosthesis in order to create an escape way for the fluid into the subgaleal space, which promotes the adhesions between the soft tissues and the prosthesis. This helps in maintaining enough supply of blood to the overlying scalp and also reduces the chances of any epidural hematoma. Patch test must be compulsory to rule out any allergic reactions to the prosthesis material., ${ }^{3,4}$

An autologous bone graft is the most ideal material to replace the soft tissues in terms of osteoactivity and biocompatibility. In cases of large cranial defects, the use of bone grafts is very limited owing to the risk of bone resorption after implantation, donor site morbidity, and infection of the bone flaps obtained from a bone bank. For decades, acrylic resin materials have been used as bone substitutes for cranial defects. In Spence's 1954 report, it is noted that there was an increased interest for acrylic resins among neurosurgeons for cranial implants owing to its simple method of fabrication at the time of surgery with auto-PMMA (polymethylmethacrylate). Acrylic implants are dimensionally stable, easy to shape, light weight, inexpensive, nonconductive, and radiolucent. A cranial prosthesis fabricated from a heat-polymerized acrylic resin before surgery has the benefits of not exposing the tissues to the heat of polymerization and residual monomers. In an attempt to make a prosthesis
${ }^{1}$ Department of Prosthodontics, Crown and Bridge, People's College of Dental Sciences and Research Centre, Bhopal, Madhya Pradesh, India

${ }^{2}$ Department of Prosthodontics, RajaRajeswari Dental College and Hospital, Bengaluru, Karnataka, India

Corresponding Author: Sunil K Mishra, Department of Prosthodontics, Crown and Bridge, People's College of Dental Sciences and Research Centre, Bhopal, Madhya Pradesh, India, Phone: +91 7697738478, e-mail: sunilmsr200@yahoo.co.in

How to cite this article: Mishra SK, Chowdhary R. Prosthetic Rehabilitation of Cranial Defects: An Overview. Int J Prosthodont Restor Dent 2019;9(2):33-34.

Source of support: Nil

Conflict of interest: None

radiopaque, gutta percha points were incorporated in the final prosthesis. The major disadvantage with PMMA is its infection rate of approx. $5 \%$ mainly in patients with a history of simultaneous orbital infections and patients who had cranial reconstructions with a history of previous infections. In growing children, PMMA had limited use owing to its inert behavior and does not adapt to the changing craniofacial skeleton. Polyether ether ketone (PEEK) is a recently introduced material for cranioplasty, but its efficacy in cranial prosthesis is still a matter of research. Room temperature vulcanizing (RTV) silicone material was also used for the fabrication of the cranial prosthesis owing to its flexibility, lifelike appearance, and good surface texture. As face is the forefront of esthetics, so these prostheses can be easily shade-matched, which improves the esthetics of the patients and enhances the social acceptance. ${ }^{3,5-7}$

Titanium is already a well-established material for the fabrication of cranial prosthesis. It is a highly biocompatible material with very rare inflammatory reactions and low corrosion and toxicity rate. The key advantage with titanium is its natural osseointegration factor, which helps in promoting active bone growth onto the implant surface. The Young's modulus of titanium is higher than that of natural bone which reduces the stress shielding. Titanium prosthesis requires prefabrication, which increases the cost and time. Intraoperative alteration of the prosthesis is often difficult and, owing to its high conductivity, discomfort may occur to patients in warmer climatic conditions. Titanium sheets of $0.61 \mathrm{~mm}$ thickness are adequate, which permit radiodensity in most radiographic studies. The tissue acceptance of the prosthesis is enhanced by anodization in a $10 \%$ sulfuric acid, $80 \%$ phosphoric acid, and $10 \%$ water solution. ${ }^{3,8}$

Mopkar et al. ${ }^{2}$ had rehabilitated a patient with a large frontal cranial defect with a heat-polymerized PMMA acrylic resin prosthesis. Mathew et al. ${ }^{7}$ had reported a case of cranioplasty on the right forehead region following a surgical excision of tumor

(c) The Author(s). 2019 Open Access This article is distributed under the terms of the Creative Commons Attribution 4.0 International License (https://creativecommons. org/licenses/by-nc/4.0/), which permits unrestricted use, distribution, and non-commercial reproduction in any medium, provided you give appropriate credit to the original author(s) and the source, provide a link to the Creative Commons license, and indicate if changes were made. The Creative Commons Public Domain Dedication waiver (http://creativecommons.org/publicdomain/zero/1.0/) applies to the data made available in this article, unless otherwise stated. 
and rehabilitated with an RTV silicone prosthesis. Blake et al. ${ }^{8}$ had reported a case of a blowout fracture with the loss of the right frontal bone. In this case, the patient was rehabilitated with an acrylic prosthesis but he was dissatisfied with the appearance; therefore, after 3 years, titanium cranioplasty was done. Gupta et al. ${ }^{9}$ had presented a case of 15-year-old boy who had suffered extensive loss of the right cranium following a road traffic accident. The patient required rehabilitation of the right frontotemporal cranial anatomy and was managed using a custom-made heat-polymerized acrylic alloplastic implant. Joseph et al. ${ }^{3}$ had reported a case of a large bony defect on the left side of the skull with no sensorineural dysfunctions and rehabilitated it with CAD/CAM system with a titanium prosthesis.

Recently, CAD/CAM-generated cranial prostheses have been introduced, which simplify the restoration of complex cranial defects, with reduction in time for intraoperative adjustments and reduce the risk of contamination. This prosthesis had its limitations owing to increased cost and nonavailability at many centers. In CAD/ CAM techniques, the imaging of the patients' cranial defect is done, and a PMMA/titanium prosthesis is made using a three-dimensional (3D) printer. 3D computed tomography (CT) and stereolithographic models evaluate the exact volume and contour of the defect and thus ensure the exact fit of the prosthesis.

\section{References}

1. Abdulai A, Iddrissu M, et al. Cranioplasty using polymethyl methacrylate implant constructed from an alginate impression and wax elimination technique. Ghana Med J 2006;40(1):18-21.

2. Mopkar MA, Aras MA, et al. Prosthetic rehabilitation of a large frontal cranial defect-A case report. J Clin Diagn Res 2018;12(11): ZD03-ZD05.

3. Joseph TM, Ravichandran R, et al. Prosthetic rehabilitation in neurosurgical cranioplasty. J Indian Prosthodont Soc 2018;18(1): 76-81. DOI: 10.4103/0972-4052.246674.

4. Wiltshire WA, Ferreira MR, et al. Allergies to dental materials. Quintessence Int 1996;27(8):513-520.

5. Neumann A, Kevenhoerster K. Biomaterials for craniofacial reconstruction. GMS Curr Top Otorhinolaryngol Head Neck Surg 2009;8:1-17. DOI: 10.3205/cto000060.

6. Spence WT. Form-fitting plastic cranioplasty. J Neurosurg 1954; 11(3):219-225. DOI: 10.3171/jns.1954.11.3.0219.

7. Mathew $\mathrm{N}$, Joseph $\mathrm{S}$, et al. Rehabilitation of a craniofacial defect using extracranial Prosthesis. J Int Oral Health 2016;8(7):813-816.

8. Blake GB, Mac Farlane MR, et al. Titanium in reconstructive surgery of the skull and face. Br J Plast Surg 1990;43(5):528-535. DOI: 10.1016/ 0007-1226(90)90115-G.

9. Gupta L, Aparna IN, et al. Cranioplasty with custom-made alloplastic prosthetic implant: A case report. World J Clin Cases 2014;2(9): 482-487. DOI: 10.12998/wjcc.v2.i9.482. 